\title{
A Low Complexity Active Interference Cancellation Method for OFDM based Cognitive Radios
}

\author{
Rammoorthy D \\ Robotics Division, CVRDE \\ DRDO \\ Chennai, India \\ sdram149@yahoo.com
}

\author{
Murugesa Pandian P.A., Srikanth S \\ Communications Lab \\ AU-KBC Research Center \\ Chennai, India \\ srikanth@au-kbc.org
}

\begin{abstract}
We propose a low complexity active interference cancellation method applicable for OFDM based cognitive radios. The motivation for the new method comes from existing techniques proposed in the literature. We compare the performance of the new method with existing technique by using simulation results to highlight the interference suppression performance, power efficiency, and complexity.
\end{abstract}

Cognitive Radio, OFDM, Low complexity Iterative AIC

\section{INTRODUCTION}

Cognitive radios (CRs) are considered to be promising for various wireless applications in the light of the severe spectrum shortages [1]. Specifically, military systems are likely to be the early adopters of CRs due to various reasons. Orthogonal frequency division (OFDM) is considered as a suitable technology for use in $\mathrm{CR}$ due to the presence of certain inherent components like FFT and IFFT in the radio blocks. However, there are important challenges in getting CR systems to work in the presence of other legacy users (LUs) transmission. These include fast detection of the LUs transmission, bandwidth, etc., even at low SNR values. In this paper we focus on an OFDM CR working in the presence of a LU transmission. We shall address the interference caused by the OFDM CR to the LU transmission assuming that we can detect the latter and its bandwidth. In section II, we discuss an important method presented in the literature for reducing interference in the LU's band by a CR. We present a low complexity method for achieving the same objective in section III. In section IV, we present detailed simulation results followed by conclusions.

\section{ACTIVE INTERFERENCE CANCELLATION}

In an OFDM based CR, interference should be minimized in the LU band for co-existence purposes. To achieve this, active interference cancellation (AIC) has been proposed in [2] for a UWB based multiband OFDM system. Consider $N$ subcarriers (tones) in the OFDM system as shown below in fig.1. Data is sent on the subcarriers adjacent to the LU band so that the interference from the CR is minimized in the LU band. In [3], details of the MMSE solution have been discussed along with simulation results. The number of adjoining subcarriers used denoted by AICn and the bandwidth of the LU in terms of the subcarriers denoted by $L U_{n}$ are varied in the study. Since the MMSE solution involves an inverse calculation, the complexity of the solution can be prohibitive as we increase $A I C_{n}$ and $L U_{n}$. A low complexity solution which depends only on $A I C_{n}$ has been proposed in [4].

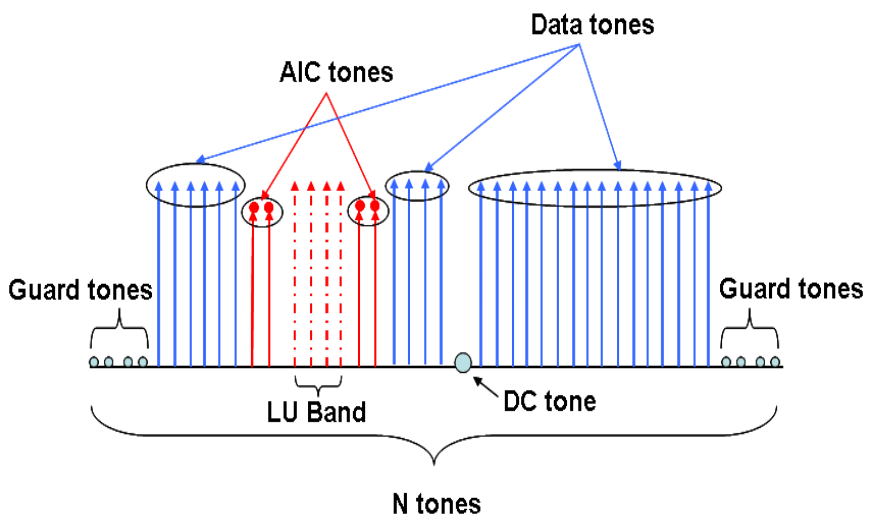

Figure 1. Subcarrier Usage in a OFDM based CR

We present the main points in the mathematical model considered in [3] for further discussions. Let $F_{d_{1} X d_{2}}=\left\{F_{n, m}\right\}$ be the $\mathrm{d}_{1}$ point FFT matrix with elements $F_{n, m}=\exp \left(\frac{-j 2 \pi n m}{d_{1}}\right)$. The time domain samples of the OFDM symbol can be expressed as,

$$
x=\frac{1}{N} F_{N X N}^{H} X
$$

Where $X=\left[X_{1}, X_{2}, \ldots \ldots X_{n}\right]^{T}$ is the data symbols fed as an input to the IFFT in the OFDM transmitter and ()$^{\mathrm{H}}$ is the Hermitian operation. Since we would like to measure the interference caused be the CR in the LU band at the higher resolution, we oversample the spectrum of the transmitted signals as,

$$
S=F_{v N X N} x=Q X
$$

Where $Q=\frac{1}{N} F_{v N X N} F_{N X N}^{H}$, and $v$ is the spectrum oversampling factor. Let the LU band span from a subcarrier 
index $i$ to $i+L U_{n}-1$, where $i$ indicates the starting position of the LU band which can be flexible. We assume that the CR will not transmit any data on these subcarrier positions. The data to be transmitted on the $A I C_{n}$ tones is given by the MMSE solution in [3] as,

$$
X_{I}=-\left(Q_{I}^{H} Q_{I}\right)^{-1} Q_{I}^{H} Q_{B} X_{B}
$$

where $Q_{B}$ is the subset of $Q$ containing only the rows corresponding to the LU band, $\mathrm{Q}_{\mathrm{I}}$ is the subset of $\mathrm{Q}_{\mathrm{B}}$ containing only the columns corresponding to subcarriers where the CR data is transmitted, $\mathrm{X}_{\mathrm{B}}$ is the $\mathrm{CR}$ data vector with zeros in the cancellation and LU band subcarriers positions and $(.)^{\mathrm{H}}$ represents the Hermitian transpose of a matrix.

Simulation results in [3] indicate the effectiveness of this solution. However, we present further results which can be used for improving performance. To measure the effectiveness of the AIC method, we consider the average interference over the LU band. We have studied the interference suppression performance for different values of $L U_{n}$ and $A I C_{n}$. Results shown in Fig. 2 indicate that for a given $L U_{n}$, increasing $A I C_{n}$ above a certain value leads to increasing interference. The extra power consumed due to the use of AIC tones increases with $L U_{n}$ and $A I C_{n}$ as shown in Fig.3. The reason for the increased interference above a certain value of AIC tones is that the matrix product, $\mathrm{Q}^{\mathrm{H}} \mathrm{Q}$, becomes ill conditioned, for a given value of licensed user band $L U_{n}$. The inversion of such an ill conditioned matrix makes the MMSE solution diverge from the optimum. We have to use pseudo inverse (pinv in MATLAB) of the $\mathrm{Q}^{\mathrm{H}} \mathrm{Q}$. The results obtained by using such a pseudo inverse are shown in Fig. 4 and 5. The performance numbers from the plots are better, but in finding the pseudo inverse, the convergence of the singular values and its singular vectors is the main concern. The convergence is very slow for a large size ill conditioned matrix [5].

\section{LOW COMPLEXITY METHOD}

We propose an iterative method to the above problem which will lead to lesser complexity while achieving good interference performance. The low complexity iterative AIC (LIAIC) is based on splitting $A I C_{n}$ into smaller values called as $A I C_{i t r}$ and finding MMSE solutions for the smaller problems. For example, given a $A I C_{n}$ value, we split it into several $A I C_{i t r}$ values and find an MMSE solution for $A I C_{i t r}$ tones at each step. We stop when we have a solution for the $A I C_{n}$ tones. The choice of $A I C_{i t r}$ for a given $A I C_{n}$ can be made based on complexity and performance aspects. The steps in LIAIC are as follows:

i. Initialize $\mathrm{Q}$ and $\mathrm{Q}_{\mathrm{B}}$ matrix for given system specifications namely number of subcarriers $N, L U_{n}$, $A I C_{n}$, and $v$.

ii. Determine the number of iterations to be performed for LIAIC tones calculation. Use the $A I C_{n}$ and $A I C_{i t r}$ values to obtain the number of iterations. Note that the $A I C_{i t r}$ value need not be the same in all iterations.

iii. Identify the submatrix $\mathrm{Q}_{\mathrm{I}}$ from $\mathrm{Q}_{\mathrm{B}}$ by considering only the columns corresponding to the AIC tones farthest from the centre of LU band, with $A I C_{i t r} / 2$ number of tones on either side of the LU band.

iv. Compute the LIAIC tone values by using the eqn 3 .

v. Update the above computed tone values at their corresponding position in $\mathrm{X}_{\mathrm{B}}$ vector.

vi. Repeat steps from iii to vi by suitably selecting the $\mathrm{Q}_{\mathrm{I}}$ submatrix corresponding to the AIC tone positions converging towards the $\mathrm{LU}$ band, in steps of $A I C_{i t r}$ number of tones, till all the values of AIC tones are computed

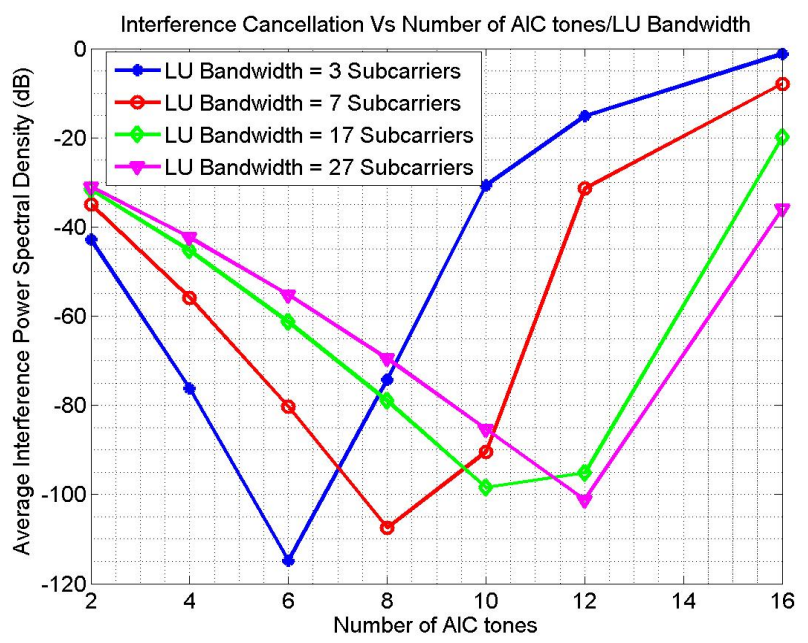

Figure 2. Interference cancellation performance of AIC technique

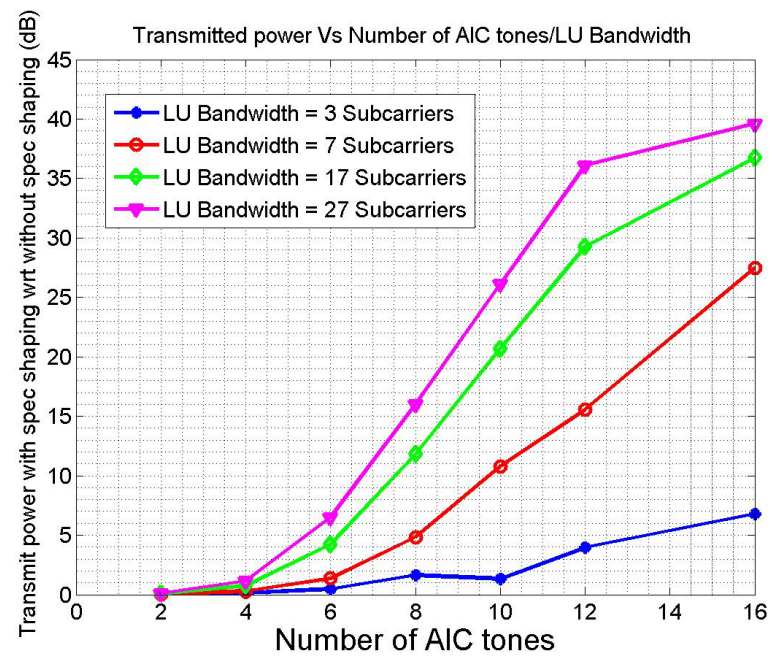

Figure 3. Performance in terms of power tranmistted by AIC technique

We need not use the same value of $A I C_{i t r}$ in all iterations. However, the sum of the $A I C_{i t r}$ should equal $A I C_{\mathrm{n}}$.

By splitting $A I C_{n}$ into smaller values of $A I C_{i t r}$ in multiple iterations, the size of the $\mathrm{Q}^{\mathrm{H}} \mathrm{Q}$ is reduced in all iterations and thereby the complexity in singular vector convergence is reduced. 


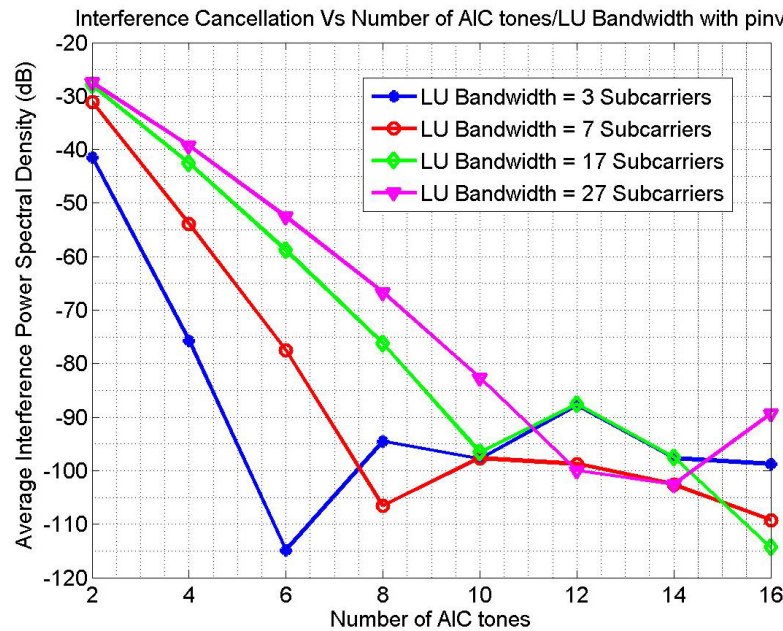

Figure 4. Interference cancellation performance of AIC technique with pseudo inverse

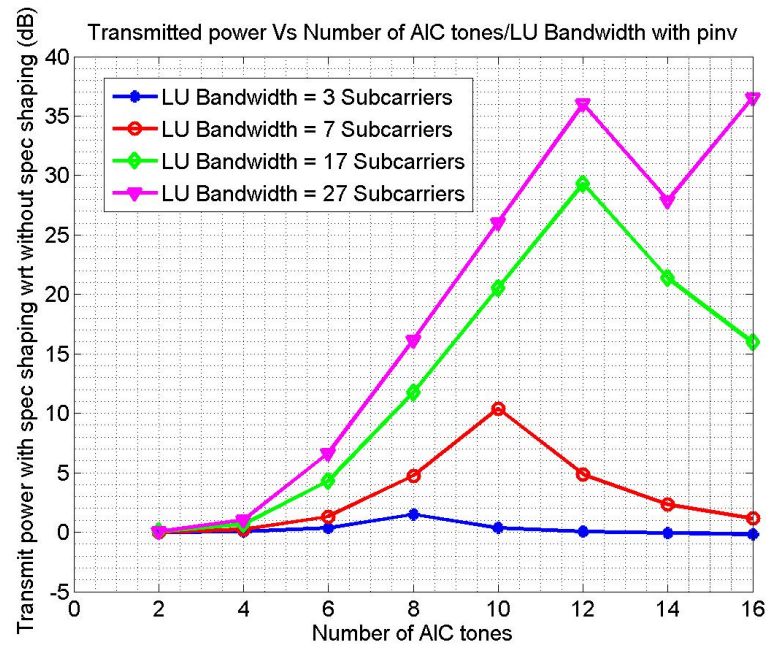

Figure 5. Performance in terms of power tranmistted by AIC technique with pseudo inverse

\section{Simulation Results}

We compare the performance of the AIC method in [3] with that of the LIAIC method. Simulations were done with similar parameters as in [3], i.e., $\mathrm{N}=256, L U_{n}$ values of 3, 7, 17 , and 27, and BPSK symbols in the data tones. For comparison, the comparative plots of the LIAIC and the AIC methods are shown in figures 6 and 7 respectively. Only the case with $L U_{n}=17$ and $A I C_{n}=12$ is considered in these figures. The set of combinations considered for LIAIC is $\{(4$, $4,4),(6,6),(8,4)$, and $(10,2)\}$. The $\mathrm{x}$ axis integer values in all these 3 plots refer to the index of the combination in the above set. For example, the 4 th combination in the set refers to $(10,2)$ combination of $A I C_{i t r}$. These plots clearly highlight the advantage of LIAIC and the tradeoff between complexity, power efficiency and the interference suppression performance.

From figures 6 and 7, in the LIAIC method, the average interference level value and the relative power transmission value are $-108.27 \mathrm{~dB}$ and $26.11 \mathrm{~dB}$ respectively. These values obtained by using the $A I C_{i t r}$ combination as $(8,4)$, with the index 3 in the above discussed $A I C_{i t r}$ combination set. The same interference level value in the AIC method is possible with $A I C_{n}=16$, as shown in Fig.4, leading to increased complexity. However this complexity gain in the LIAIC comes with increased relative power transmission. Thus, there is a tradeoff between the power transmitted and the complexity. This means the LIAIC method can result in better complexity compared to the AIC method.

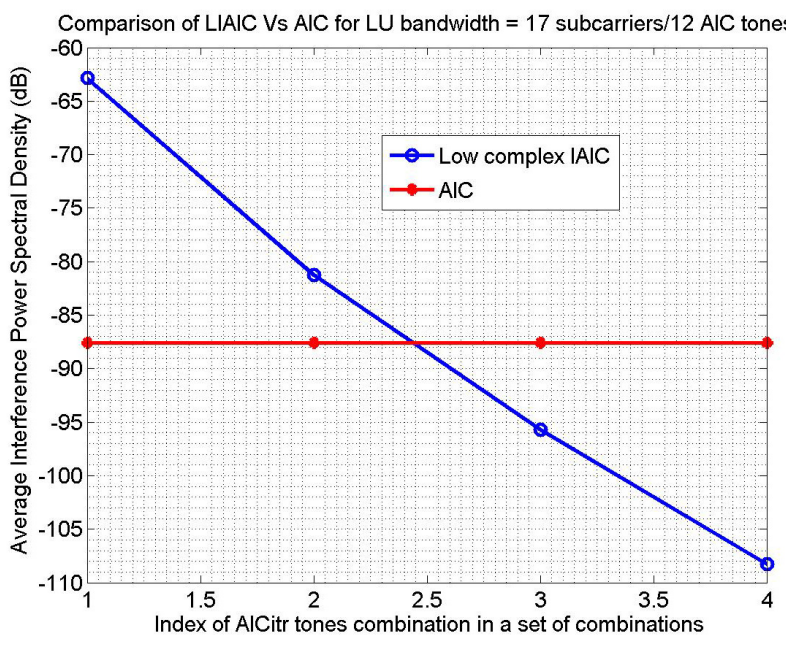

Figure 6. Interference Cancellation: Comparison between LIAIC and AIC

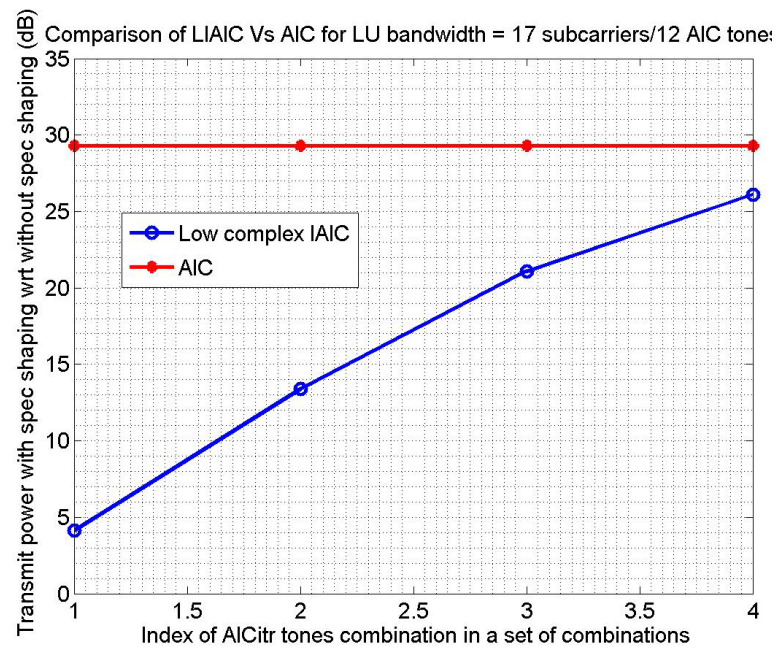

Figure 7. Transmitted Power: Comparison between LIAIC and AIC

\section{CONCLUSION}

We have proposed the LIAIC method for superior performance in OFDM based CRs. Under certain conditions, the LIAIC method can outperform the AIC method in complexity aspects. Extensive simulation results have been presented to highlight the tradeoffs that can be used judiciously in the LIAIC based CRs. 


\section{REFERENCES}

[1] Hüseyin Arslan, "Cognitive Radio, Software Defined Radio,And Adaptive Wireless Systems", Signals and Communication Technology, Springer publication.

[2] Hirohisa Yamaguchi, "Active interference cancellation technique for MBOFDM cognitive radio," in 34th European Microwave Conference, 2004,vol. 2, pp. 1105-1108.

[3] Hisham A. Mahmoud and H"useyin Arslan,"Spectrum Shaping of OFDM-based Cognitive Radio Signals", IEEE Radio and Wireless Symposium, 2008, 22-24 Jan. 2008, Page(s):113 - 116.

[4] Shih-Gu Huang and Chien-Hwa Hwang, "Low Complexity Active Interference Cancellation for OFDM Cognitive Radios", WCNC 2008, Pages 1279-1283.

[5] Gibert Strang, "Linear Algebra and its Applications", fourth edition, Cengage learning 